\title{
Cystic Vestibular Schwannomas and Post-Gamma Knife Radiosurgery-Induced Necrosis of Vestibular Schwannomas: Report of Two Cases and Review of Literature
}

\author{
Reddy Ravikanth ${ }^{1}$ Pooja Majumdar ${ }^{2}$ Denver Steven Pinto ${ }^{1}$ \\ ${ }^{1}$ Department of Radiology, Holy Family Hospital, Thodupuzha, \\ Idukki District, Kerala, India \\ 2Department of Medicine, Indian Naval Hospital Ship Kalyani, \\ Gandhigram PO, Visakhapatnam, Andhra Pradesh, India \\ Address for correspondence Reddy Ravikanth, MD, DNB, \\ Department of Radiology, Holy Family Hospital, Thodupuzha, Idukki \\ District, Kerala 685605, India (e-mail: ravikanthreddy06@gmail.com).
}

Asian J Oncol 2020;6:44-46

\begin{abstract}
Cystic vestibular schwannomas (VS) form a rare subgroup that differs from the solid variant clinically and radiologically. Certain unique features reported in cystic VS

Keywords

- cerebellopontine angle

- GKRS

- vestibular

schwannomas are larger size, presentation with atypical initial symptoms such as dysgeusia, vertigo, facial pain and gait unsteadiness, short duration of symptoms, rapid progression of symptoms or sudden deterioration and frequent involvement of facial nerve. Gamma Knife radiosurgery (GKRS) is a safe and effective treatment for VS, achieving tumor control and resulting in a very low morbidity rate. Here, we described two cases of cystic vestibular schwannomas prior to GKRS and their radiological appearances post-GKRS.
\end{abstract}

\section{Case Report}

A 76-year-old female presented with gradual left-sided hearing loss and impaired cerebellar function tests. Magnetic resonance imaging (MRI) revealed a cystic left cerebellopontine angle lesion causing significant mass effect on adjacent cerebellum, pons, and cerebellar peduncle with vasogenic edema in the ipsilateral cerebellum. Left vestibulocochlear, facial nerve complex root entry zone (origin), is masked by

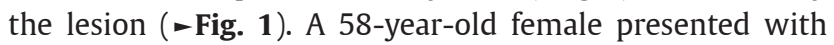
gradual hearing loss and left-sided tinnitus. MRI revealed marked peripheral enhancement of the left cerebellopontine angle lesion with central nonenhancing areas (-Fig. 2). A 51-year-old female presented with vertigo, facial weakness, and hearing loss emerging 6 weeks following Gamma Knife radiosurgery (GKRS) of a right-sided vestibular schwannoma (VS). Brain MRI demonstrated central nonenhancement within the schwannoma, likely related to radiation necrosis (-Figs. 3 and $\mathbf{4}$ ).

\section{Discussion}

VSs account for approximately 8 to $10 \%$ of primary intracranial tumors and $80 \%$ of cerebellopontine angle tumors. ${ }^{1}$
These tumors may undergo cystic changes, frequently as microcysts, caused by degenerative changes within the tumors. Occasionally, these areas may coalesce to form larger cysts. Piccirillo et $\mathrm{al}^{2}$ classified cystic VS as follows: type A-central and thick-walled cysts which can be further subdivided as (1) polycystic (multiple small intratumoral cysts with thick-walled cyst), (2) polycystic (multiple moderate-sized intratumoral cysts with thick-walled cyst), and (3) monocystic (single-large cyst); and type B-peripheral and thin-walled cysts which can be further classified as (1) anterior, (2) medial, (3) posterior, and (4) combined. It has also been found that a cystic VS is less responsive to Gamma Knife surgery than a solid counterpart. ${ }^{3}$ In conclusion, cystic VS are a heterogeneous group presenting with short duration and rapid progression of symptoms with frequent facial nerve involvement due to sudden expansion of the cystic elements. VSs are the most common tumors of the cerebellopontine angle (CPA), accounting for more than 90\% of all such tumors. ${ }^{4}$ VS are typically benign, slow-growing tumors. Symptoms at diagnosis commonly include hearing deficiency or loss, tinnitus, loss of balance, and, more rarely, change in facial sensation and headache. Diagnosis is performed by MRI showing a typical gadolinium-enhanced lesion. The principal management options for VS are watchful waiting, 


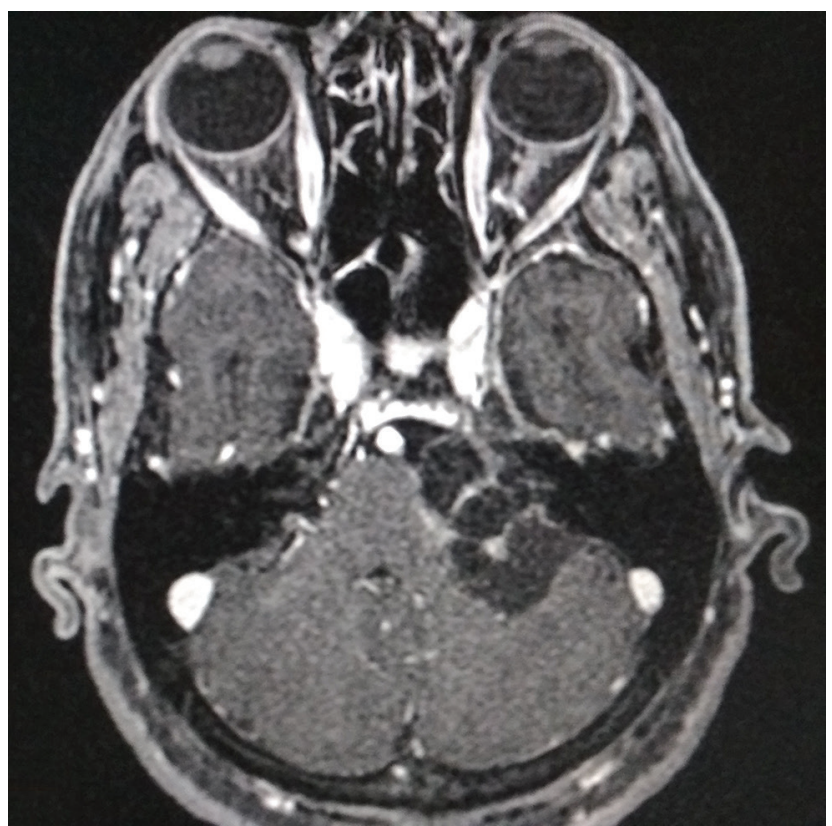

Fig. 1 Axial three-dimensional fat-suppressed magnetic resonance imaging with contrast of a 76-year-old female patient demonstrating a left-sided type-1B (Piccirillo's classification) cystic vestibular schwannoma (star) causing significant mass effect on adjacent cerebellum, pons and cerebellar peduncle. Left vestibulocochlear, facial nerve complex root entry zone (origin), is masked by the lesion.

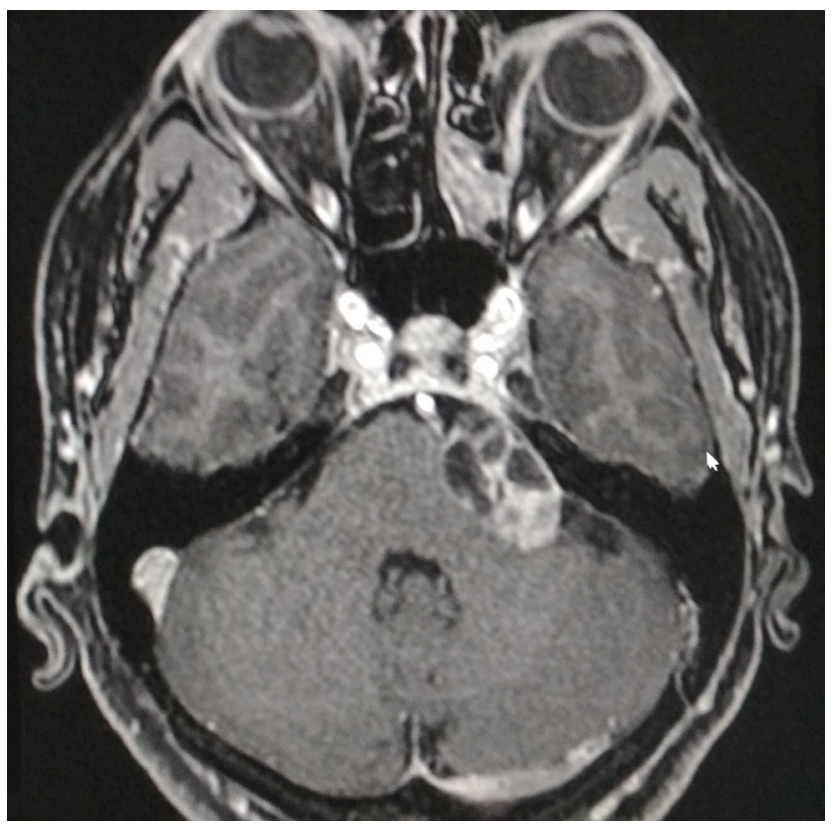

Fig. 2 Axial three-dimensional fat-suppressed magnetic resonance imaging with contrast of a 58-year-old female patient who presented with a left-sided tinnitus demonstrating a left sided type-1A (Piccirillo's classification) cystic vestibular schwannoma (star) causing mass effect on the pons, cerebellar peduncle and VII-VIII nerve complex.

surgery, and radiotherapy. The goal of any intervention is to maximize tumor control while limiting damage to the nearby cranial nerves, brainstem, vascular structures, and cochlea. GKRS has been shown to provide excellent tumor control for VS, with rates upwards of $97 \%$ at 10 years posttreatment. ${ }^{5}$

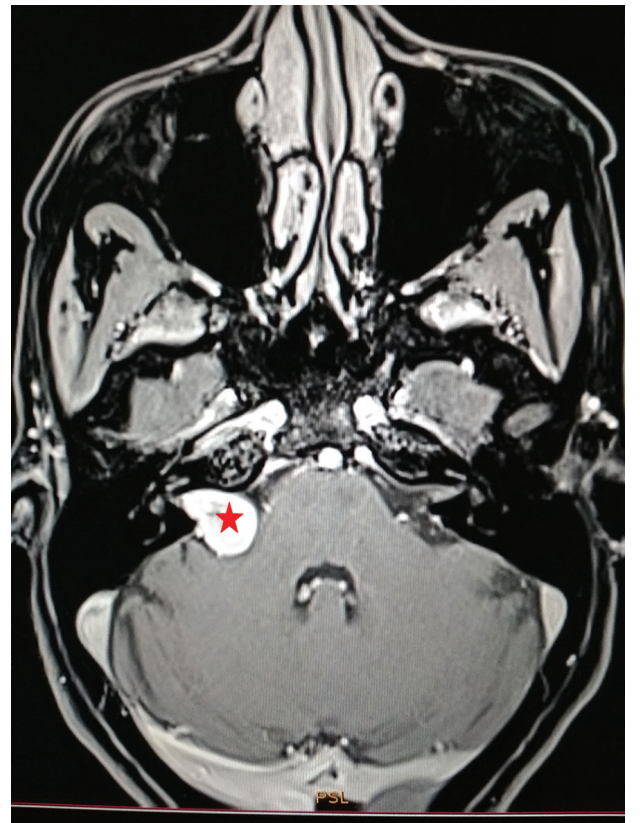

Fig. 3 Pre-GKRS axial volumetric interpolated breath-hold examination fat-suppressed magnetic resonance imaging image obtained in a 51-year-old female with tinnitus and imbalance demonstrating a right cerebellopontine angle vestibular schwannoma (star). GKRS, Gamma Knife radiosurgery.

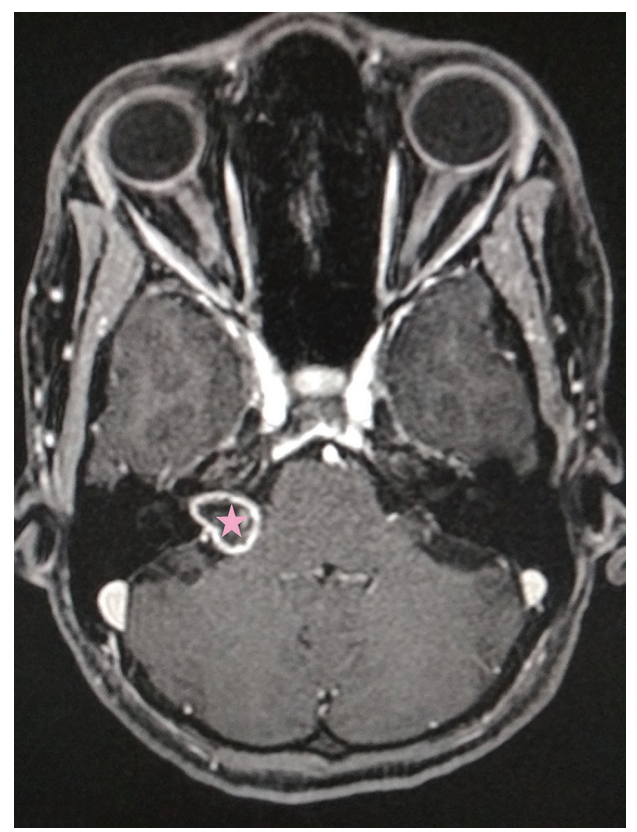

Fig. 4 Post-GKRS axial three-dimensional fat-suppressed magnetic resonance imaging image obtained 6 weeks later demonstrating a rim of peripheral enhancement with central necrosis in a right cerebellopontine angle vestibular schwannoma (star). GKRS, Gamma Knife radiosurgery.

Clinical studies by Yang et al, which utilized GKRS for the treatment of large VS (diameter greater than $3 \mathrm{~cm}$ ), revealed worse tumor control rates and higher patient morbidity as compared with small tumors (diameter $\leq 3 \mathrm{~cm}$ ). ${ }^{6}$ Based on the aforementioned studies, neurosurgeons and radiation 
oncologists must take tumor size, growth rate, and location, as well as the patient's neurological function and age, into account when determining the ideal course of action for a VS. Large tumors causing significant brainstem and cranial nerve compression, or those that have already failed radiosurgery require surgical intervention. GKRS is a safe and effective treatment for VS, achieving tumor control and resulting in a very low morbidity rate. Few complications reported with GKRS are cysts with multiple septae developing medial to the tumor causing compression of the brain stem and fourth ventricle, thus necessitating post-GKRS surgery. ${ }^{7}$ Multiple cranial neuropathies of the cerebellopontine angle after GKRS should raise suspicion of necrosis within the schwannoma and urgent MRI should be considered.

\section{Conflict of Interest}

None declared.

\section{References}

1 Benech F, Perez R, Fontanella MM, Morra B, Albera R, Ducati A. Cystic versus solid vestibular schwannomas: a series of 80 grade III-IV patients. Neurosurg Rev 2005;28(3):209-213

2 Piccirillo E, Wiet MR, Flanagan S, et al. Cystic vestibular schwannoma: classification, management, and facial nerve outcomes. Otol Neurotol 2009;30(6):826-834

3 Unger F, Walch C, Haselsberger K, et al. Radiosurgery of vestibular schwannomas: a minimally invasive alternative to microsurgery. Acta Neurochir (Wien) 1999;141(12):1281-1285; discussion 1285-1286

4 Lunsford LD, Niranjan A, Flickinger JC, Maitz A, Kondziolka D. Radiosurgery of vestibular schwannomas: summary of experience in 829 cases. J Neurosurg 2005;102(Suppl):195-199

5 Williams BJ, Xu Z, Salvetti DJ, McNeill IT, Larner J, Sheehan JP. Gamma Knife surgery for large vestibular schwannomas: a single-center retrospective case-matched comparison assessing the effect of lesion size. J Neurosurg 2013;119(2):463-471

6 Yang HC, Kano H, Awan NR, et al. Gamma Knife radiosurgery for larger-volume vestibular schwannomas. Clinical article. J Neurosurg 2011;114(3):801-807

7 Iwai Y, Yamanaka K, Ishiguro T. Surgery combined with radiosurgery of large acoustic neuromas. Surg Neurol 2003;59(4):283-289, discussion 289-291 